\title{
Word Distance Affects Subjective Temporal Distance
}

\author{
Cheng Wang, Yu Liu and Jun Wang* \\ Department of Psychology, Zhejiang Normal University, Jinhua, China
}

The kappa effect is a well-reported phenomenon in which spatial distance between discrete stimuli affects the perception of temporal distance demarcated by the corresponding stimuli. Here, we report a new phenomenon that we propose to designate as the lexical kappa effect in which word distance, a non-magnitude relationship of discrete stimuli that exists in the lexical space of the mental lexicon, affects the perception of temporal distance. A temporal bisection task was used to assess the subjective perception of the time interval demarcated by two successively presented words. Word distance was manipulated by varying the semantic (Experiment 1) or phonological (Experiment 2) similarity between the two words. Results showed that the temporal distance between the two words was perceived to be shorter when the

\section{OPEN ACCESS}

Edited by:

Xiaolin Zhou,

Peking University, China

Reviewed by:

Peter Dixon

University of Alberta, Canada

Walter Gerbino,

University of Trieste, Italy

Xinlin Zhou,

Beijing Normal University, China

*Correspondence:

Jun Wang

jun.wang@zjnu.edu.cn

Specialty section:

This article was submitted to

Language Sciences,

a section of the journal

Frontiers in Psychology

Received: 04 October 2021 Accepted: 12 November 2021 Published: 15 December 2021

Citation:

Wang C, Liu Y and Wang J (2021) Word Distance Affects Subjective

Temporal Distance.

Front. Psychol. 12:785303. doi: 10.3389/fpsyg.2021.785303 corresponding two words were lexically closer. We explain this effect within the internal clock framework by assuming faster detection of the word that terminated timing when it is preceded by a semantically or phonologically similar word.

Keywords: word distance, time perception, temporal bisection task, lexical kappa effect, internal clock

\section{INTRODUCTION}

The perception of time is a universal, continuous experience, which is central to virtually all behaviors (Paton and Buonomano, 2018). Literatures show that the subjective duration of a given stimulus can be influenced by many non-temporal physical factors, such as spatial size, weight, moving velocity, luminance, and loudness of the stimulus (see Winter et al., 2015; Matthews and Meck, 2016; for reviews). Among these physical factors, one of particular relevance is spatial distance, whose influence on time perception has been demonstrated in the well-known "kappa effect". Generally speaking, the kappa effect is such that when two brief flashes are successively presented at different locations, increasing the spatial distance between the two locations results in longer perceived temporal distance between the two flashes (Cohen et al., 1953; Price-Williams, 1954). This effect has also been demonstrated in auditory (Grondin and Plourde, 2007; Sarrazin et al., 2007) and tactile (Suto, 1952; Grondin et al., 2011) modalities. An influential account of this effect is the theory of magnitude (ATOM, Walsh, 2003; Bueti and Walsh, 2009; but see Jones and Huang, 1982 for another account) which assumes that time, space, number, and other dimensions are processed using a common magnitude system. The magnitude of space can bias the perceived magnitude of time because the perceptions of both dimensions are represented by the same magnitude system. The kappa effect shows the dependence of perceived time on spatial distance, which is a magnitude in our physical world. Here, we report a new phenomenon that time perception is affected by word distance in the mental lexicon, which is a non-magnitude distance that exists in our psychological world. 
Mental lexicon is a mental store of words that contains information regarding words' meaning, pronunciation, spelling, and syntactic characteristics (Elman, 2004; Castro and Stella, 2019). It plays a central role in language comprehension and production (Aitchison, 2012; Wang and Zhang, 2021). Models of mental lexicon assume that the information stored in it is structured as a large-scale network consisting of nodes (or vertices) that correspond to words and connections (or edges) that represent the relations between two words (Collins and Loftus, 1975; Steyvers and Tenenbaum, 2005; De Deyne et al., 2017). The connection between words is a multiplexed system, with different layers of relations based on semantic similarity (taxonomic or associative relation, e.g., dog-cat, dog-hunt) or phonological similarity (word-form overlapping, e.g., dog-log) (Stella et al., 2018; Castro and Stella, 2019). Networks based on semantic (Steyvers and Tenenbaum, 2005; De Deyne et al., 2017) or phonological (Coleman, 1998; Carlson et al., 2014) connections, or both connections simultaneously (Stella et al., 2018; Castro and Stella, 2019) have been successfully used to model various language phenomena.

The activation spreading model (Collins and Loftus, 1975) of mental lexicon assumes that activation spreads in the lexical network from one node to others, and the spreading activation dissipates with distance. The distance between the nodes (i.e., word distance) is determined by the degree of semantic or phonological similarity between the words, such that word distance decreases as word similarity increases (Collins and Loftus, 1975; den Heyer and Briand, 1986; Prabhakaran et al., 2014). For example, the node representing the word dog will be closer to the node representing the word cat or $\log$ than to the node representing the word cup. The model predicts that when a node is activated, close nodes will receive larger activation than distant ones, thereby facilitating the processing of the words represented by the closer nodes. Accordingly, a number of studies reported that presenting a prime word (e.g., dog) facilitated comprehending a target word (e.g., cat or log) that had semantic or phonological similarities to the prime word (Hillinger, 1980; Rouibah et al., 1999; Zhou and Marslen-Wilson, 2000). These findings of semantic and phonological priming effects demonstrated that the effects of word distance could be obtained by manipulating semantic and phonological similarities.

The current study aims to investigate whether the word distance in the mental lexicon affects time perception. Two words were successively presented briefly, and participants were asked to judge the temporal distance between the two words as long or short. Word distance between the two words were varied such that the two words were either close or distant, via manipulating semantic (Experiment 1) or phonological (Experiment 2) similarity (Hillinger, 1980; Rouibah et al., 1999; Zhou and Marslen-Wilson, 2000). We used the classic temporal bisection task (Penney et al., 2008; Matthews and Meck, 2016; Li et al., 2021) to examine the subjective temporal distance. In this task, participants are first presented with multiple "short" and "long" anchor time intervals, followed by a test phase in which they judge a set of intermediate intervals (aka, the probe intervals) as being closer to the "short" or "long" anchor intervals. Behavior performances were then fit to a psychometric curve to estimate the point of subjective equality (PSE). Larger PSEs indicate shorter perceived time (see "Materials and Methods" section for details). If the "kappa effect" exists also in the lexical space of the mental lexicon, we expect to observe that the temporal distance would be perceived to be shorter (i.e., larger PSEs) when it is flanked by a pair of close words than when flanked by a pair of distant words.

\section{MATERIALS AND METHODS}

\section{Participants}

We conducted an a priori power analysis using $\mathrm{G}^{*}$ Power (Faul et al., 2007) to determine the necessary sample size. This analysis (two dependent means, cohen's $d=0.5$, alpha $=0.05$, power $=$ 0.8 , two tails) gave a minimum sample size of 34 participants. Experiment 1 (15 males, mean age $22.50 \pm 3.28$ years) and Experiment 2 (12 males, mean age $21.65 \pm 2.71$ years) each recruited 34 participants. For each experiment, we collected data until 34 participants met our inclusion criteria. Three and four participants were replaced in Experiments 1 and 2, respectively, following the exclusion criteria described in the "Data Analysis" section. All participants were native speakers of Mandarin Chinese, had normal hearing and normal or correctedto-normal vision. All participants gave written informed consent before the experiment and received monetary compensation. This study was approved by the Research Ethics Committee of Zhejiang Normal University.

\section{Material}

We used word similarity to manipulate word distance (Close vs. Distant) in the lexical network of mental lexicon (Collins and Loftus, 1975; den Heyer and Briand, 1986; Prabhakaran et al., 2014). Two conditions of word pairs were used. In the Close condition the two words in a pair had semantic (Experiment 1) or phonological (Experiment 2) similarity, while in the Distant condition the two words had no similarity. All words were Chinese two-character words. First, 25 words were selected as primes. Then, each prime word was paired with two target words. One of them had similarity with the prime (the Close condition), and the other had no similarity with the prime (the Distant condition). In Experiment 1, the pair of prime and target words in the Close condition had a semantic similarity (taxonomically related, i.e., belonging to the same category) but no other similarity, for example, 樱桃(“cherry”,/ying1tao2/) 一草莓(“strawberry”,/cao3mei2/). In Experiment 2, the pair of prime and target words in the close condition had a phonological similarity (their first characters had the same pronunciation) but no other similarity, e.g., 梳子(“comb”,/shu1zi5/)——书展(“book fair”,/shu1zhan3/). Taxonomic relation (Ouyang et al., 2019; Rose et al., 2019) and phonological overlapping (Zhou and MarslenWilson, 2000; Zhu et al., 2015) were abundantly used to define semantic and phonological similarities, respectively, in language studies. In both experiments, the pair of prime and target words had no similarity, e.g., 樱桃一老虎(“tiger”,/lao3hu3/), and 梳子一海豚(“dolphin”,/hai3tun2/). The target words were matched between the two conditions on word frequency $(|t| s<1.398$, 
ps $>0.175)$ and number of strokes $(|t| s<1.417, p s>0.169)$. Note that we did not employ a priming paradigm, and naming the two words in a pair as prime and target is just for the convenience of description.

\section{Procedure}

We used the temporal bisection task to examine the subjective temporal distance. The temporal distance was defined as the time interval demarcated by two successively presented Chinese twocharacter words (font: Microsoft YaHei; RGB: 192192 192; visual angle: $\left.1.3^{\circ} \times 1.3^{\circ}\right)($ see Figure 1$)$. The two words each appeared $50 \mathrm{~ms}$, and the time interval separating them varied from 300 to $800 \mathrm{~ms}$. The short and long anchor intervals were 300 and $800 \mathrm{~ms}$, and the probe intervals were linearly spaced from 300 to $800 \mathrm{~ms}$ in steps of $100 \mathrm{~ms}$ (i.e., 300, 400, 500, 600, 700, and $800 \mathrm{~ms}$ ). All stimuli were presented against a black background, on a 24 -inch LED monitor (resolution: $1,920 \times 1,080$; refresh rate: $100 \mathrm{~Hz}$ ) at a viewing distance of $60 \mathrm{~cm}$.

The temporal bisection task was programmed in E-prime (Psychology Software Tools, Sharpsburg, PA, United States). This task included a training phase followed by a testing phase. In the training phase, after clear instruction about whether the subsequently presented anchor intervals would be "long" or "short" (e.g., "Next are long intervals"), each of the anchor intervals (as demarcated by successive brief presentations of “米米”) was successively presented three times. To confirm that participants could differentiate the two anchor intervals, each anchor interval was then presented three times with a randomized order, and participants were asked to categorize them as "long" or "short." Participants passed the training phase only after making $100 \%$ correct responses. Those who made errors would receive another round of training phase. In the testing phase, each trial (see Figure 1) began with a fixation $(+)$ at the center of the screen for $500 \mathrm{~ms}$, followed by a blank screen for $200 \mathrm{~ms}$. After that, two words were successively presented, each for $50 \mathrm{~ms}$. The interval separating the two words was selected from the probe intervals (i.e., 300, 400, 500, 600, 700, and $800 \mathrm{~ms}$ ).
Then, after a blank of $300 \mathrm{~ms}$, a question mark appeared at the center of the screen for 4,000 ms. Participants were asked to judge, upon seeing the question mark, whether the time interval between the two words as being closer to the "long" or "short" anchor interval. The next trial began $500 \mathrm{~ms}$ after participant making response. Each of the six probe intervals was presented with each of the 26 pairs of words for each condition (Close and Distant), resulting 300 trials. Trial order was randomized across participants. These trials were administered in two blocks that were separated by a break. Each block consisted of 150 trials and 4 warm-up trials. Each experiment lasted approximately $25 \mathrm{~min}$.

\section{Data Analysis}

For each participant, the rate of "long" judgment was calculated for each probe interval for each condition. The observed distribution of responses was fit to a psychometric logistic function (Treutwein and Strasburger, 1999). PSE was then calculated based on the $50 \%$ point in the obtained logistic curve. Larger PSEs indicate shorter perceived time. Figure 2A shows this process for an exemplary individual. Individual rates at each probe interval were averaged across participants, and submitted to the same fitting procedure to produce the grand average fitted curve, as shown in Figures 2B, 3B. If any data of an individual were unable to fit with a logistic function $\left(R^{2}<0.85\right)$, all data from this individual were discarded.

\section{RESULTS}

\section{Experiment 1}

In the exemplary individual shown in Figure 2A, the distribution of response was clearly more left-shifted in the Distant than Close conditions, resulting in a smaller PSE in the Distant condition. Individual and mean PSEs for each condition and condition differences are shown by the violin plots in Figure 2C. Importantly, paired $t$-tests revealed that PSE was significantly smaller in the Distant condition $(M=520 \mathrm{~ms}, s d=41 \mathrm{~ms})$

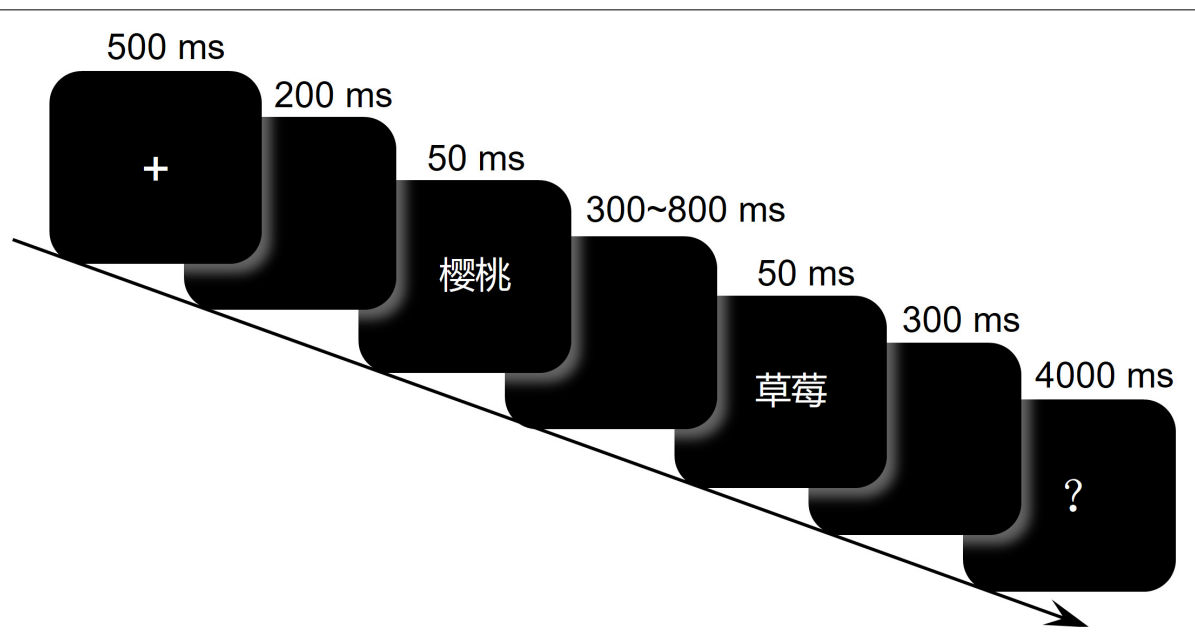

FIGURE 1 | Schematic of procedure. 

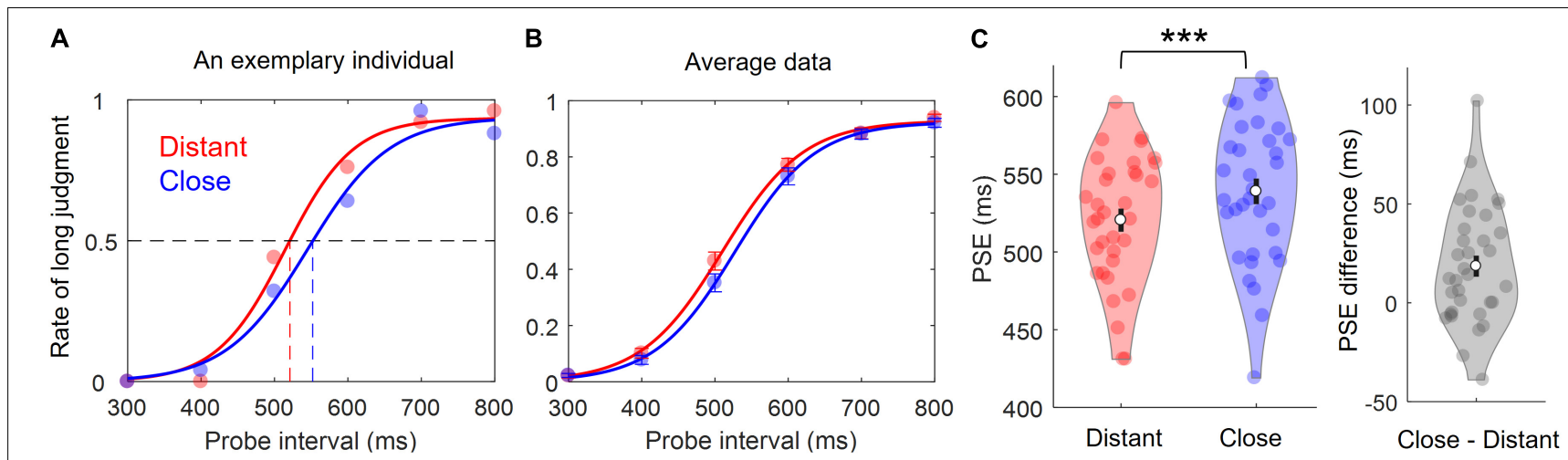

FIGURE 2 | Results of Experiment 1. (A) Individual's rates of "long" judgment as function of probe intervals (300-800 ms) in the Distant and Close conditions. A logistic curve was fit to each individual's distribution of responses to derive an estimation of the point of subjective equality (PSE). (B) Logistic curves fit to grand mean rates of "long" judgment. Dots represents mean, and error bars indicate \pm SEM. (C) Violin plots for the PSE. Colored dots represent individual data points. White dots represent averages. Error bars indicate \pm SEM. ${ }^{* \star *} p<0.001$.

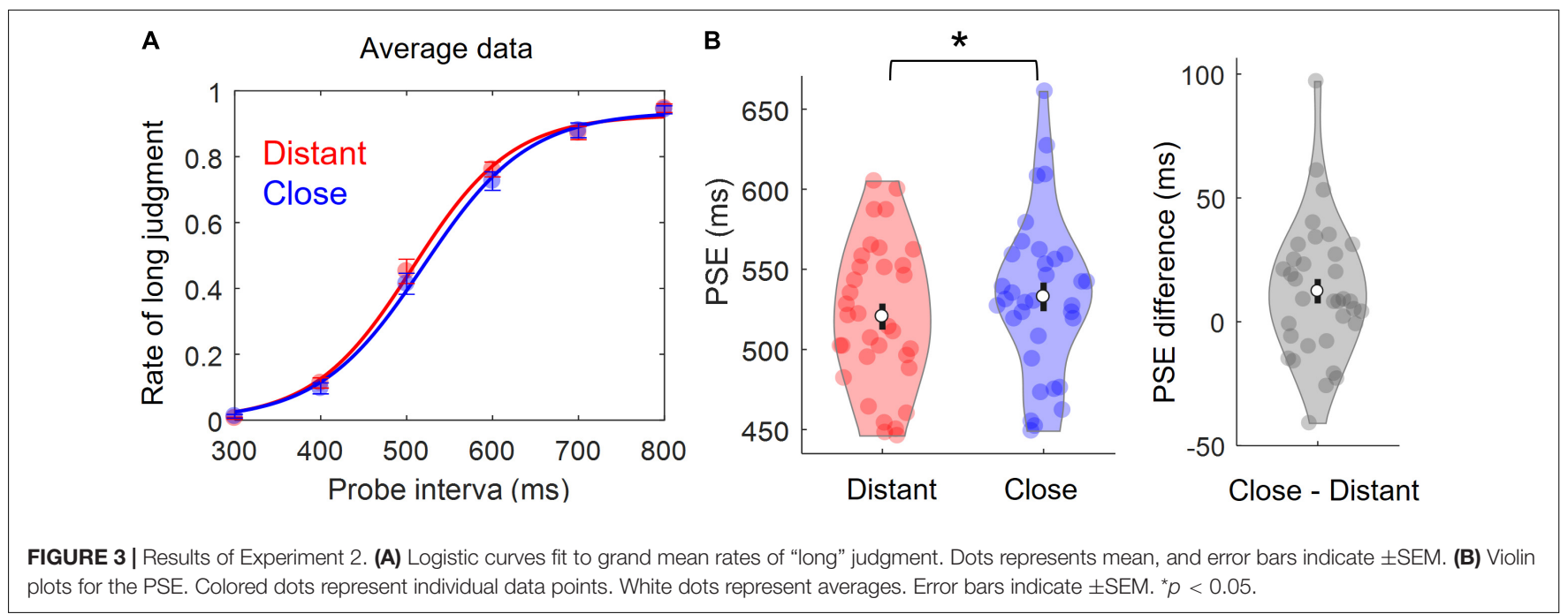

than in the Close condition $(M=539 \mathrm{~ms}, s d=46 \mathrm{~ms}), t_{(33)}=$ $-3.66, p<0.001$, Cohen's $d=0.628$. These results indicated that the temporal distance between two successively presented words was judged to be shorter in the Close than Distant conditions, and that the semantic similarity between the two words compressed time perception.

\section{Experiment 2}

As shown in the average data in Figure $\mathbf{3 A}$, the distribution of responses was clearly more left-shifted in the Distant than Close conditions, resulting in a smaller PSE in the Distant condition. Individual and mean PSEs for each condition and condition differences are shown by the violin plots in Figure 3B. Importantly, paired $t$-tests revealed that PSE was significantly smaller in the Distant condition $(M=521 \mathrm{~ms}, s d=45 \mathrm{~ms})$ than in the Close condition $(M=533 \mathrm{~ms}, s d=50 \mathrm{~ms}), t_{(33)}=$ $-2.64, p=0.013$, Cohen's $d=0.453$. These results indicated that the temporal distance between two successively presented words was judged to be shorter in the Close than Distant conditions, and that the phonological similarity between the two words compressed time perception.

To assess whether the effects of word distance on temporal distance were different across the two experiments, we conducted a $2 \times 2$ mixed-design ANOVA with type of word distance (semantic vs. phonological) as a between-subject factor and word distance (close vs. distant) as a within-subject factor. The results showed that only the main effect word distance was significant, $F=20.08, p<0.001, \eta_{p}^{2}=0.233$. The main effect of type $\left(F=0.09, p=0.772, \eta_{p}^{2}=0.001\right)$ and the interaction between type and word distance $\left(F=0.81, p=0.371, \eta_{p}^{2}=\right.$ 0.012 ) were both not significant. The absence of interaction indicated that the effects of word distance on temporal distance were comparable across the two types of word distance.

\section{DISCUSSION}

To investigate whether the "kappa effect" originally observed in the physical space can extend to the lexical space of the 
mental lexicon, two experiments using the temporal bisection task were conducted to examine whether the word distance affects time perception. We observed that the temporal distance between two successively presented words was perceived to be shorter when the corresponding two words were semantically or phonologically close in the mental lexicon, compared to when they were distant. These results indicate that two successively presented words appear to be temporally closer when they are lexically closer. We hereby propose to designate this new phenomenon as the lexical kappa effect, and explain this effect within the framework of internal clock models (Gibbon et al., 1984; Wearden, 2004; Treisman, 2013).

The original kappa effect showed that the temporal distance flanked by discrete stimuli was perceived to be shorter when the flanking stimuli were more closely spaced (Cohen et al., 1953; Price-Williams, 1954; Sarrazin et al., 2007). Likewise, the lexical kappa effect observed in the present study revealed that the temporal distance between the two successively presented words was perceived to be shorter when the word distance between the two flanking words was smaller (i.e., semantically or phonologically similar). Both effects show that a non-temporal distance between two stimuli flanking a temporal distance can bias the perceived duration of that temporal distance in the same direction as that of the non-temporal distance. But the non-temporal distance involved in the two effects are vastly different. In the original kappa effect the non-temporal distance is spatial distance, which is a tangible, daily-used magnitude in the physical space (Cohen et al., 1953; Price-Williams, 1954; Sarrazin et al., 2007), while in the lexical kappa effect the nontemporal distance is word distance, which is a non-magnitude, between-word relation in the lexical space of the mental lexicon.

The kappa effect is a special variant of a broader, general bias that time interval flanked by discrete stimuli is perceived to be shorter when difference between the stimuli is smaller (e.g., Cohen et al., 1953; Alards-Tomalin et al., 2014; Leboe-McGowan et al., 2021). In almost all previous studies examining this bias, the difference between the flanking stimuli were manipulated on magnitude across a variety of dimensions, including space (Cohen et al., 1953; Price-Williams, 1954; Sarrazin et al., 2007), pitch height (Shigeno, 1993; Henry and McAuley, 2009; Marty et al., 2021), object size (Xuan et al., 2007; Matthews, 2011), digital number (Alards-Tomalin et al., 2014), and color saturation (Alards-Tomalin et al., 2014). A general framework of ATOM (Walsh, 2003; Bueti and Walsh, 2009) was used to overall explain these magnitude-based biases (Alards-Tomalin et al., 2014). ATOM proposes that time, space, number, and other dimensions are processed as a common magnitude in the intraparietal sulcus of the brain. According to ATOM, the magnitude of one dimension of stimuli (e.g., spatial distance) interferes with the perceived magnitude of another (e.g., temporal distance) because the magnitude of both dimensions is represented in the same brain module.

Unlike the above studies, the lexical kappa effect is a time perception bias that was rooted in a non-magnitude dimension, i.e., the lexical space of the mental lexicon. We manipulated the difference of flanking stimuli on word distance, which was essentially semantic or phonological relatedness between words and cannot be characterized as a magnitude. Thus, our results cannot be explained by the ATOM theory, as it relies on magnitude difference of stimuli. A recent study (Leboe-McGowan et al., 2021) also excluded ATOM from explaining distorting effects from non-magnitude dimensions. In this study, stimuli flanking a time interval were two letters that either shared the same identity or not (Experiment 1), a color word and a colored rectangle that either described the same color or not (Experiment 2), or a sentence stem and a terminal word that fit either well or poorly (Experiment 3). In all cases, Leboe-McGowan et al. (2021) found that letter or conceptual overlap (i.e., smaller difference) between flanking stimuli compressed time perception. They argued that ATOM was not applicable for explaining their findings because letter and conceptual overlap were not magnitude and ATOM required shared representation of magnitude.

Instead, we attempt to explain the lexical kappa effect within the framework of internal clock models (Gibbon et al., 1984; Wearden, 2004; Treisman, 2013). According to these models, the subjective perception of time is computed by an internal clock mechanism that consists of a pacemaker, a switch and an accumulator. The pacemaker emits at a given rate pulses that flows into the accumulator, and the switch controls the gate of the pulse flow. At the onset of timing, the switch closes, allowing pulses to flow into the accumulator; at the offset of timing, the switch opens and stops the flow. The number of pulses accumulated during the event(s) being timed represents the duration of the time. The more pulses are accumulated, the longer the duration is perceived to be. In this framework, nontemporal features of stimuli can affect the latency to close or open the switch, or the pacemaker rate, thereby producing a change in its judged duration.

In the current study, the to-be-timed intervals were demarcated by two successive words. The initiating words signaled the switch to close, and the terminal words signaled the switch to open. Before participants became sensitive to the terminal words, the responses of the internal clock (i.e., latency to close switch and pacemaker rate) across the two conditions should be equivalent, because the initiating words and the display during the to-be-timed interval were identical across conditions. Thus, the effect of word distance could only be explained by different latencies to open the switch at the end of timing, which were signal by the terminal words. The terminal words had a semantic (Experiment 1) or phonological (Experiment 2) similarity with the initiating words in the Close condition, but had no similarity with the initiating words in the Distant condition. As word similarity not only facilitates word recognition (Hillinger, 1980; Rouibah et al., 1999; Zhou and Marslen-Wilson, 2000) but also increases perceptual sensitivity of words (Rhodes et al., 1993; Pastore et al., 2003), we can infer that the terminal words were detected faster in the Close condition than in the Distant condition, which would lead to relatively earlier latencies to open the switch and hence less pulses transferred into the accumulator in the Close condition. If so, it would lead participants to judge time intervals flanked by similar words as shorter. Some other studies adopted a similar explanation to explain their observations of shortening 
effect of flanking stimuli (Grondin et al., 1996; Matthews, 2011; Leboe-McGowan et al., 2021). For example, Grondin et al. (1996) observed that time intervals were judged as shorter when they were flanked by a visual signal followed by an auditory signal than when flanked by two successive visual signals, and they explained this effect by assuming that the offset of the interval was detected faster when it was marked by an auditory signal.

The faster processing of the terminal words when preceded by a semantically or phonologically similar words can be explained under the spreading activation model of the mental lexicon (Collins and Loftus, 1975). In the lexical network, word distance is shorter for similar words than for dissimilar words. When the initiating words were activated, activation spread along the network and dissipated with distance. Compared to terminal words in the Distance condition, terminal words in the Close condition were closer to the initiating words, and therefore received larger spreading activation. This could have facilitated the detection of the terminal words, and resulted in earlier latencies to open the switch (i.e., end timing) of the internal clock in the Close condition. Although the network model is prevalent, alternative models had also been proposed to explain the semantic or phonological priming effects (e.g., Masson, 1995; McRae et al., 1997; McNamara, 2005). For example, the distributed memory model (Masson, 1995) assumes that a word is represented by a specific pattern of activation of a collection of processing units, and the semantic priming effect arise from similar patterns of activation that represent the prime and target words. These alternative models can also explain faster detection of the terminal words in the Close condition, and thus do not contradict the interpretation for the observed effect of word similarity on time perception within the framework of internal clock.

In conclusion, the perception of a temporal distance can be distorted by word distance in the lexical space, in a manner that smaller distance between two words shortens the perceived temporal distance demarcated by the two words. As word

\section{REFERENCES}

Aitchison, J. (2012). Words in the Mind: An Introduction to the Mental Lexicon. Hoboken, NJ: John Wiley \& Sons.

Alards-Tomalin, D., Leboe-McGowan, J. P., Shaw, J. D. M., and Leboe-McGowan, L. C. (2014). The effects of numerical magnitude, size, and color saturation on perceived interval duration. J. Exp. Psychol. 40, 555-566. doi: 10.1037/a003 5031

Bueti, D., and Walsh, V. (2009). The parietal cortex and the representation of time, space, number and other magnitudes. Philos. Trans. R. Soc. B Biol. Sci. 364, 1831-1840. doi: 10.1098/rstb.2009.0028

Carlson, M. T., Sonderegger, M., and Bane, M. (2014). How children explore the phonological network in child-directed speech: a survival analysis of children's first word productions. J. Mem. Lang. 75, 159-180. doi: 10.1016/j.jml.2014. 05.005

Castro, N., and Stella, M. (2019). The multiplex structure of the mental lexicon influences picture naming in people with aphasia. J. Complex Netw. 7, 913-931. doi: 10.1093/comnet/cnz012

Cohen, J., Hansel, C. E. M., and Sylvester, J. D. (1953). A new phenomenon in time judgment. Nature 172, 901-901. doi: 10.1038/172901a0

Coleman, J. (1998). Cognitive reality and the phonological lexicon: a review. J. Neurolinguistics 11, 295-320. doi: 10.1016/S0911-6044(97)00014-6 distance is not a magnitude property of stimulus, this bias cannot be explained by the influential ATOM theory. Within the internal clock framework, we propose to explain it by faster detection of the word that terminated timing when it is preceded by a semantically or phonologically similar word.

\section{DATA AVAILABILITY STATEMENT}

The raw data supporting the conclusions of this article will be made available by the authors, without undue reservation.

\section{ETHICS STATEMENT}

The studies involving human participants were reviewed and approved by the Research Ethics Committee of Zhejiang Normal University. The patients/participants provided their written informed consent to participate in this study.

\section{AUTHOR CONTRIBUTIONS}

CW wrote the manuscript. CW and JW designed and supervised the research. YL conducted the experiments and analyzed the data. All authors contributed to the article and approved the final version for submission.

\section{FUNDING}

This research was supported by the China National Social Science Fund in Education (2018 general project: Neural Mechanisms of Multisensory Integration Dysfunction in Autism and Related Intervention under Multi-Modality Educational Perspective (Grant No. BBA180083).

Collins, A. M., and Loftus, E. F. (1975). A spreading-activation theory of semantic processing. Psychol. Rev. 82, 407-428. doi: 10.1037/0033-295X.82.6.407

De Deyne, S., Kenett, Y. N., Anaki, D., Faust, M., and Navarro, D. (2017). "Largescale network representations of semantics in the mental lexicon," in Big Data in Cognitive Science, ed. M. N. Jones (Milton Park: Routledge), 174-202.

den Heyer, K., and Briand, K. (1986). Priming single digit numbers: automatic spreading activation dissipates as a function of semantic distance. Am. J. Psychol. 99, 315-340. doi: 10.2307/1422488

Elman, J. L. (2004). An alternative view of the mental lexicon. Trends Cogn. Sci. 8, 301-306. doi: 10.1016/j.tics.2004.05.003

Faul, F., Erdfelder, E., Lang, A.-G., and Buchner, A. (2007). G*Power 3: a flexible statistical power analysis program for the social, behavioral, and biomedical sciences. Behav. Res. Methods 39, 175-191. doi: 10.3758/BF0319 3146

Gibbon, J., Church, R. M., and Meck, W. H. (1984). Scalar timing in memory. Ann. N. Y. Acad. Sci. 423, 52-77. doi: 10.1111/j.1749-6632.1984.tb23417.x

Grondin, S., and Plourde, M. (2007). Discrimination of time intervals presented in sequences: spatial effects with multiple auditory sources. Hum. Mov. Sci. 26, 702-716. doi: 10.1016/j.humov.2007.07.009

Grondin, S., Ivry, R. B., Franz, E., Perreault, L., and Metthé, L. (1996). Markers' influence on the duration discrimination of intermodal intervals. Percept. Psychophys. 58, 424-433. doi: 10.3758/BF03206818 
Grondin, S., Kuroda, T., and Mitsudo, T. (2011). Spatial effects on tactile duration categorization. Can. J. Exp. Psychol. 65, 163-167. doi: 10.1037/a0022785

Henry, M. J., and McAuley, J. D. (2009). Evaluation of an imputed pitch velocity model of the auditory kappa effect. J. Exp. Psychol. Hum. Percept. Perform. 35, 551-564. doi: 10.1037/0096-1523.35.2.551

Hillinger, M. L. (1980). Priming effects with phonemically similar words. Mem. Cogn. 8, 115-123. doi: 10.3758/BF03213414

Jones, B., and Huang, Y. L. (1982). Space-time dependencies in psychophysical judgment of extent and duration: algebraic models of the tau and kappa effects. Psychol. Bull. 91, 128-142. doi: 10.1037/0033-2909.91.1.128

Leboe-McGowan, L. C., Leboe-McGowan, J. P., Fortier, J., and Dowling, E. J. (2021). Non-magnitude sources of bias on duration judgements for blank intervals: conceptual relatedness of interval markers reduces subjective interval duration. Psychol. Res. doi: 10.1007/s00426-021-01482-w [Online ahead of print].

Li, F., Wang, L., Jia, L., Lu, J., Wu, Y., Wang, C., et al. (2021). The varying coherences of implied motion modulates the subjective time perception. Front. Psychol. 12:602872. doi: 10.3389/fpsyg.2021.602872

Marty, N., Marty, M., and Pfeuty, M. (2021). Relative contribution of pitch and brightness to the auditory kappa effect. Psychol. Res. 85, 55-67. doi: 10.1007/ s00426-019-01233-y

Masson, M. E. J. (1995). A distributed memory model of semantic priming. J. Exp. Psychol. Learn. Mem. Cogn. 21, 3-23. doi: 10.1037/0278-7393.21.1.3

Matthews, W. J. (2011). Can we use verbal estimation to dissect the internal clock? Differentiating the effects of pacemaker rate, switch latencies, and judgment processes. Behav. Processes 86, 68-74. doi: 10.1016/j.beproc.2010.09.006

Matthews, W. J., and Meck, W. H. (2016). Temporal cognition: connecting subjective time to perception, attention, and memory. Psychol. Bull. 142, 865907. doi: $10.1037 /$ bul0000045

McNamara, T. P. (2005). Semantic Priming: Perspectives From Memory and Word Recognition. Hove: Psychology Press.

McRae, K., de Sa, V. R., and Seidenberg, M. S. (1997). On the nature and scope of featural representations of word meaning. J. Exp. Psychol. 126, 99-130. doi: 10.1037/0096-3445.126.2.99

Ouyang, M., Cai, X., and Zhang, Q. (2019). The effect of lexical cohort size is independent of semantic context effects in a picture-word interference task: a combined ERP and sLORETA study. Front. Hum. Neurosci. 13:439. doi: 10. 3389/fnhum.2019.00439

Pastore, R. E., Crawley, E., Skelly, M. A., and Berens, M. S. (2003). Signal detection theory analyses of semantic priming in word recognition. J. Exp. Psychol. Hum. Percept. Perform. 29, 1251-1266. doi: 10.1037/0096-1523.29.6.1251

Paton, J. J., and Buonomano, D. V. (2018). The neural basis of timing: distributed mechanisms for diverse functions. Neuron 98, 687-705. doi: 10.1016/j.neuron. 2018.03.045

Penney, T. B., Gibbon, J., and Meck, W. H. (2008). Categorical scaling of duration bisection in pigeons (Columba livia), mice (Mus musculus), and humans (Homo sapiens). Psychol. Sci. 19, 1103-1109. doi: 10.1111/j.1467-9280.2008. 02210.x

Prabhakaran, R., Green, A. E., and Gray, J. R. (2014). Thin slices of creativity: using single-word utterances to assess creative cognition. Behav. Res. Methods 46, 641-659. doi: 10.3758/s13428-013-0401-7

Price-Williams, D. R. (1954). The Kappa effect. Nature 173, 363-364. doi: 10.1038/ $173363 \mathrm{a} 0$

Rhodes, G., Parkin, A. J., and Tremewan, T. (1993). Semantic priming and sensitivity in lexical decision. J. Exp. Psychol. Hum. Percept. Perform. 19, 154-165. doi: 10.1037/0096-1523.19.1.154

Rose, S. B., Aristei, S., Melinger, A., and Abdel Rahman, R. (2019). The closer they are, the more they interfere: semantic similarity of word distractors increases competition in language production. J. Exp. Psychol. Learn. Mem. Cognit. 45, 753-763. doi: $10.1037 / \mathrm{xlm} 0000592$
Rouibah, A., Tiberghien, G., and Lupker, S. J. (1999). Phonological and semantic priming: evidence for task-independent effects. Mem. Cogn. 27, 422-437. doi: 10.3758/BF03211538

Sarrazin, J.-C., Giraudo, M.-D., and Pittenger, J. B. (2007). Tau and Kappa effects in physical space: the case of audition. Psychol. Res. 71, 201-218. doi: 10.1007/ s00426-005-0019-1

Shigeno, S. (1993). The interdependence of pitch and temporal judgments by absolute pitch possessors. Percept. Psychophys. 54, 682-692. doi: 10.3758/ BF03211792

Stella, M., Beckage, N. M., Brede, M., and De Domenico, M. (2018). Multiplex model of mental lexicon reveals explosive learning in humans. Sci. Rep. 8:2259. doi: 10.1038/s41598-018-20730-5

Steyvers, M., and Tenenbaum, J. B. (2005). The large-scale structure of semantic networks: statistical analyses and a model of semantic growth. Cogn. Sci. 29, 41-78. doi: $10.1207 / s 15516709 \operatorname{cog} 2901 \_3$

Suto, Y. (1952). The Effect of Space on Time Estimation (S-ffect) in Tactual Space. I. Japanese J. Psychol. 22, 45-57.

Treisman, M. (2013). The information-processing model of timing (Treisman 1963): its sources and further development. Timing Time Percept. 1, 131-158.

Treutwein, B., and Strasburger, H. (1999). Fitting the psychometric function. Percept Psychophys. 61, 87-106.

Walsh, V. (2003). A theory of magnitude: common cortical metrics of time, space and quantity. Trends Cogn. Sci. 7, 483-488. doi: 10.1016/j.tics.2003. 09.002

Wang, C., and Zhang, Q. (2021). Word frequency effect in written production: evidence from ERPs and neural oscillations. Psychophysiology 58, e13775. doi: 10.1111/psyp. 13775

Wearden, J. H. (2004). Decision processes in models of timing. Acta Neurobiol. Exp. 64, 303-317.

Winter, B., Marghetis, T., and Matlock, T. (2015). Of magnitudes and metaphors: explaining cognitive interactions between space, time, and number. Cortex 64, 209-224. doi: 10.1016/j.cortex.2014.10.015

Xuan, B., Zhang, D., He, S., and Chen, X. (2007). Larger stimuli are judged to last longer. J. Vis. 7, 2-2. doi: 10.1167/7.10.2

Zhou, X., and Marslen-Wilson, W. (2000). The relative time course of semantic and phonological activation in reading Chinese. J. Exp. Psychol. Learn. Mem. Cogn. 26, 1245-1265. doi: 10.1037/0278-7393.26.5. 1245

Zhu, X., Damian, M. F., and Zhang, Q. (2015). Seriality of semantic and phonological processes during overt speech in Mandarin as revealed by eventrelated brain potentials. Brain Lang. 144, 16-25. doi: 10.1016/j.bandl.2015. 03.007

Conflict of Interest: The authors declare that the research was conducted in the absence of any commercial or financial relationships that could be construed as a potential conflict of interest.

Publisher's Note: All claims expressed in this article are solely those of the authors and do not necessarily represent those of their affiliated organizations, or those of the publisher, the editors and the reviewers. Any product that may be evaluated in this article, or claim that may be made by its manufacturer, is not guaranteed or endorsed by the publisher.

Copyright $\odot 2021$ Wang, Liu and Wang. This is an open-access article distributed under the terms of the Creative Commons Attribution License (CC BY). The use, distribution or reproduction in other forums is permitted, provided the original author(s) and the copyright owner(s) are credited and that the original publication in this journal is cited, in accordance with accepted academic practice. No use, distribution or reproduction is permitted which does not comply with these terms. 